\title{
Inhomogeneous Measurement High-speed Reconstruction Method of Im- age Block Compressed Sensing
}

\author{
Cheng Zhou ${ }^{1}$ and Yanhong $\mathrm{Lu}^{2, *}$ \\ ${ }^{I}$ Hubei Key Laboratory of Intelligent Wireless Communications, South-Center University for Nationalities, Hubei Wu- \\ han 430074, China \\ ${ }^{2}$ Wuhan Polytechnic, Wuhan, 430077, China
}

\begin{abstract}
The complexity of block compressed sensing of images algorithm is very high. And it is too difficult to apply the algorithm on hardware platform. A low complexity compressed sensing method based on non-uniform measurement is presented in this paper which use different measurement to the transform domain coefficient with different significance of every image blocks. And as the experimental results on PC platform suggest, the proposed method is better than original method on reconstructed image's subjective and objective quality. And this method is optimized and implemented on a high performance digital signal processor TMS320C6455 platform including algorithm structure design and C code optimization according to the hardware feature. It is also faster than original method and it get about 4 times speedup on the DSP platform after optimization. The proposed method can be combined with the existing image and video compression scheme very easily.
\end{abstract}

Keywords: Image compressed sensing, digital signal processor; optimized implementation, random measurement, image reconstruction.

\section{INTRODUCTION}

In recent years, with rapid development of information technology including internet and high-speed mobile communication network, the demand of the user for transmitting, storing and sharing image signal is higher and higher [1]. There is no doubt that image and video compression technology is the key technology to solve image/video data storage and transmission [2]. Image and video compression technology is developing rapidly, and there are more and more emerging techniques and new standards are emerging $[3,4]$, but the technical principle still can't break away from traditional compression framework of image spatial domain Nyquist sampling, transformation, quantization and entropy coding. With the improvement of performance of acquisition device, image and video resolution increases exponentially, and more and more static images and video images with high definition needs efficient compression and storage transmission. Therefore, how to break through the traditional compression framework becomes the research hotspot.

Compressed sensing theory is the emerging signal processing theory in recent years $[5,6]$. The theory points out if a signal meets scarcity, the signal can be sampled by setting an effective non-full rank measurement matrix, or the signal can be sampled with sampling frequency which is lower than Nyquist frequency, and the measured value is used to solve optimization problem to reconstruct original signals accurately. There have been wide literatures indicating the validity of the theory in the field of image and video compression [7], which is an opportunity breaking through traditional Nyquist sampling framework. But the present researches focus on theoretical simulation and verification in various fields, and there are few literatures about design and implementation of practical system. Compression sensing method needs lots of matrix and iterative operations, the hardware resource consumption is more and the implementation cost is higher, which makes it difficult to implement and popularize hardware system.

TMS320C6455 is a new fixed-point digital signal processor which is launched by Texas Instruments Company. The highest dominant frequency is $1.2 \mathrm{GHz}$, and it is a common DSP with high monokaryon performance [8]. It has the characteristics of low power consumption and high processing capacity. And it has evident advantage for size and cost. For typical block image compression sensing framework [9], the paper analyzes the part which is complicated and difficult to realize hardware, and proposes to improve the framework. With the help of capability of TMS320C6455 DSP processing powerful signals, the paper designs and implements a high-speed image compression sensing measurement scheme with low complexity, which not only can efficiently complete image compression sampling and restore reconstruction, but also can fully reduce the difficulty of realizing image compression sensing in hardware system. 


\section{ANALYSIS ON IMAGE COMPRESSED SENSING FRAMEWORK}

\subsection{Measurement and Reconstruction of Compressed Sensing}

According to the basic theory of compressed sensing, if original signal or transformation-domain signal is sparse, a few of measured values can be used to reconstruct original signal. The measurement evidently reduces the sampling rate of Nyquist sampling theorem. The scarcity of signal can be indicated by the following example.

If a real signal $z \in R^{N}$ with the length of $\mathrm{N}$ can be expressed by the linear combination of a group of standard orthogonal basis $\Phi$ (the coefficient of orthogonal basis is $\left.\phi_{i}, i=1,2 \ldots N\right)$.

$z=\sum_{i=1}^{N} x_{i} \varphi_{i}=\Phi \mathbf{x}$

$\mathrm{X}$ and $\mathrm{z}$ is the vector with the length of $\mathrm{N}$. If the expression of signal $\mathrm{z}$ on orthogonal basis $\Phi$ only has $k \leq N$ non zero coefficients, $\Phi$ is sparse base of signal z, the sparsity is $\mathrm{k}$, and the signal $\mathrm{z}$ is the signal which can meet sparsity or which can be compressed.

In the measurement process of compressed sensing, the compressed signals are projected on measurement matrix to achieve measured value, and the process can be expressed by the following formula.

$\mathbf{y}=\Psi_{z}=\Psi \Phi x=\Theta x$

$\boldsymbol{\Psi}$ is $M \times N$ measurement matrix, and $\mathrm{M}$ is generally less than N. $\boldsymbol{\Theta}=\boldsymbol{\Psi} \boldsymbol{\Phi}$ is sensing matrix, y is the signal value after measurement, and the length is $\mathrm{M}$. The dimension $\mathrm{M}$ of the achieved measurement value $\mathrm{Y}$ is less than the dimension $\mathrm{N}$ of the compressed signal $\mathrm{x}$. Candes and Tao proved if the measurement matrix meets restricted isometric property, it can ensure $\mathrm{x}$ with the length of $\mathrm{N}$ can restore $\mathrm{y}$ with the length of $M \geq O(k \log N)$ [5]. Holger proved that when “ $\boldsymbol{\Psi}$ " is a Gaussian randomized matrix, sensing matrix $\boldsymbol{\Theta}$ can meet restricted isometric condition with large probability [10], so Gaussian random matrix is generally used as measurement matrix $\boldsymbol{\Psi}$. Based on the above measurement process, we can get that signal sampling frequency of compressed sensing is lower than Nyquist sampling frequency, which breaks through the restriction of Nyquist sampling theorem. $\mathrm{M} / \mathrm{N}$ is generally defined as the key parametermeasurement ratio in compressed sensing algorithm. The present theoretical researches focus on if better reconstruction quality can be achieved under low measurement ratio.

In the reconstruction process of compressed sensing signals, $M=N$ and $\mathbf{y}=\boldsymbol{\Theta x}$ is an underdetermined problem, so there are infinite solutions. As $x$ meets scarcity, $x$ can be reconstructed in y by solving minimum $l_{0}$ norm of the following formula. $\hat{x}=\arg \min \|x\|_{0}$ s.t. $\Theta x=y$

It is very difficult to solve the minimum $l_{0}$ norm. When the measurement matrix $\boldsymbol{\Psi}$ has no relationship with sparse basis $\Phi$ [11], the minimum $l_{0}$ norm can be converted to be the minimum $l_{1}$ norm, and the formula (3) is converted to be formula (4).

$\hat{x}=\arg \min \|x\|_{1}$ s.t. $\Theta x=y$

Lastly, the original signal $\mathrm{z}$ can be reconstructed by $\hat{z}=\Phi \hat{x}$.

From the above analysis, we can know that the measurement value y includes the information of original signal z. If it meets the conditions, the original signal can be reconstructed by formula (4). But the in the measurement process of signals, there is noise interference, rounding error and quantization error. Under the condition of allowing distortion of reconstructing signals, formula (4) can be transformed to be formula (5) for solution.

$\hat{x}=\arg \min \|x\|_{1}$ s.t. $\|\Theta x-y\|_{2}<\varepsilon$

When the error between reconstructed signal $\hat{x}$ and compressed signal $\mathrm{x}$ is less than a constant, the reconstruction is considered to be successful. Therefore, the reconstruction algorithm of compressed sensing system is a problem solving optimization. The typical reconstruction algorithm in image compressed sensing system is Orthogonal Matching pursuit [12]. OMP algorithm can be reconstructed under the condition of given iterative times, the computation complexity and reconstruction quality is balanced, which is suitable for the reconstruction of image signals with large data quantity. The system in the paper uses OMP algorithm.

\subsection{Framework of Block Image Compressed Sensing}

The data volume of natural image is huge, so a gray image with $512 \times 512$ resolution needs $10^{6 \text { orders }}$ of magnitude of matrix for storage. The transformation-domain signals of the image are directly sampled, large matrix operation in the transformation and measurement process consumes more computation resources. And the computational complexity of reconstruction algorithm increases. Gan proposed Block Compressed Sensing method [8]. After the images are divided into image blacks with small size, the images are transformed, measured and reconstructed, and the computational complexity reduces, which realizes performance compromise. In fact, the basic idea of the method is similar to that of traditional image/video compression, which avoids block matrix operation of images with large size. Natural image signals can't meet scarcity in space, so the original images receive compressed sensing measurement in transformation domain, and the framework of the algorithm is shown in Fig. (1).

In the above chapter, the paper proves that the size of block of BCS method determines the computational 

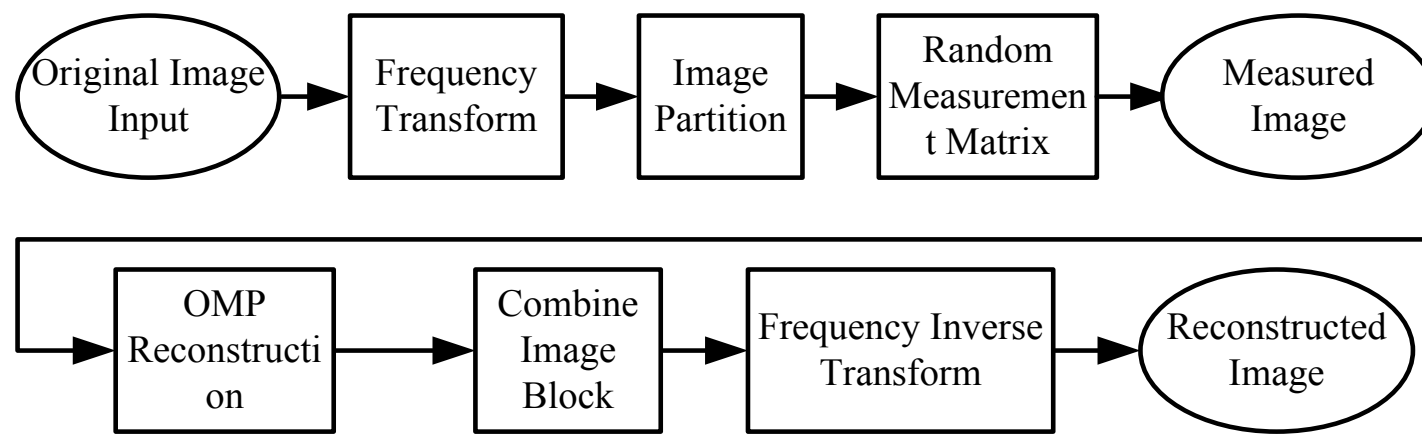

Fig. (1). Typical compressed sensing processing method block images.

Table 1. Experiment results of Lenna image under BCS method.

\begin{tabular}{|c|c|c|c|}
\hline $\begin{array}{c}\text { Block } \\
\text { Size }\end{array}$ & $\begin{array}{c}\text { Measurement } \\
\text { Rate }\end{array}$ & $\begin{array}{c}\text { Reconstructed } \\
\text { Image PSNR(dB) }\end{array}$ & $\begin{array}{c}\text { Time-consuming } \\
\text { on PC (s) }\end{array}$ \\
\hline \hline $32 \times 32$ & 0.5 & 30.64 & 86.70 \\
\hline $16 \times 16$ & 0.5 & 30.44 & 16.32 \\
\hline $8 \times 8$ & 0.5 & 28.86 & 4.51 \\
\hline $4 \times 4$ & 0.5 & 11.94 & 2.76 \\
\hline
\end{tabular}

complexity and reconstruction quality of the image compressed sensing system [13]. Matlab software is used to simulate compressed sampling and reconstruction process of a Lenna gray image with the resolution ration of $512 \times 512$ on PC platform. The platform is configured to be WIN7, intel I5-3470 CPU and 4GB. Under the condition that the size of image is $32 \times 32,16 \times 16,8 \times 8$ and $4 \times 4$, the simulation result when the measurement ration is 0.5 is shown in Table 1. It needs to be noticed that random matrix is different in algorithm operation, so all test results in the paper use the mean value of 10 experiments as the final experiment result. In order to make the comparison easy, the iterative times of OMP reconstruction algorithm is valued according to $1 / 4$ of sparsity. From Table 1, we can see that with the increase of blocks, the quality of BCS method reconstructing images is better, but the system consumption increases with geometrical progression. When the blocks are small, the reconstruction quality is worse, but the system consumption is little. When the size of block is $16 \times 16$ or $8 \times 8$, the reconstruction quality and system consumption concluded good balance. And it is similar to the image block size of image and video compressed framework.

\section{IMPROVED BCS METHOD}

BCS method has achieved good processing speed and performance compromise of reconstruction quality, but the characteristic that image signal energy after DCT or DWT makes transformation coefficient important is not considered, higher measurement ratio is required to achieved better reconstruction quality. For the disadvantage of $\mathrm{BCS}$, there are researchers improving the measurement strategy [14, 15]. For example, Yang pre-weights the transformation-domain coefficient by image statistics, which improves the efficiency of measurement. $\mathrm{Yu}$ proposes an image compressed sampling method based on image evidence, which improves the efficiency of measurement. But the common characteristic of these methods is to increase computational complexity based on original BCS method, which not only makes the original computational complexity more complicated than higher framework, but also makes it difficult to be implemented on hardware system. According to the disadvantages of the above background techniques, the paper proposes a nonhomogeneous compressed sensing measurement scheme without increasing computational complexity which uses unified measurement strategy for each image block to improve measurement efficiency.

The algorithms which can be implemented on hardware system hope to have the structure with higher repeatability. Especially for the condition of processing block images, the operation strategy of each block is different. If the measurement of each image block is different, the efficiency processing the hardware is low. As a matured transformation, the hardware implementation of DCT transformation is not difficult, and the important energy coefficients focus on the top right corner of transformation block. The importance of coefficients after DCT transformation is different. In the traditional image/video compression framework, the feature is used, and most non-important coefficients are quantized to be zero in the subsequent algorithm, which improves the compressibility of the following entropy coding. If the important coefficients and non-important coefficients use unified measurement strategy in the compressed sensing system, 


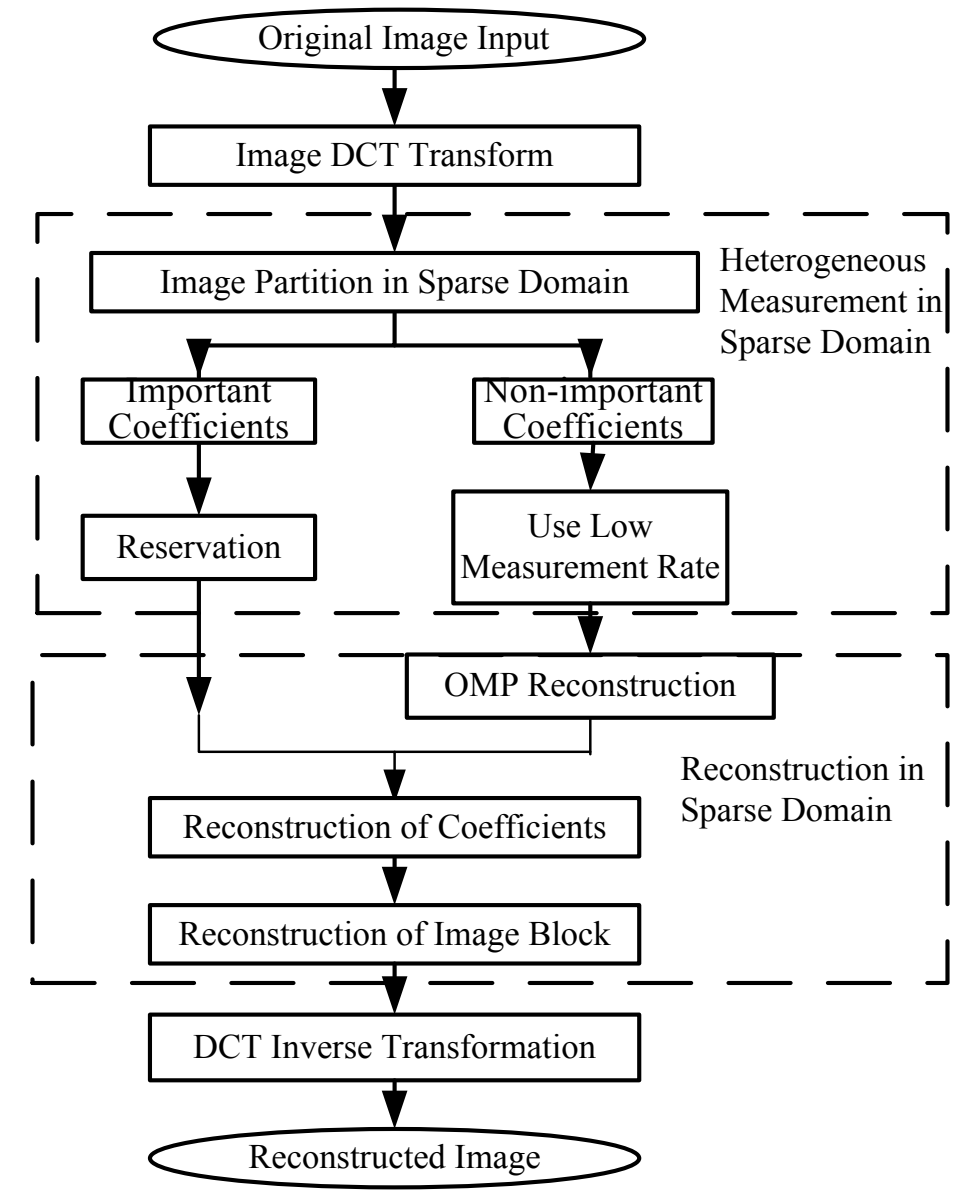

Fig. (2). Heterogeneous compressed sensing measurement method.

there is no doubt that the efficiency is low. So the coefficients after DCT transformation are divided into two types, important and non-important coefficients. The important coefficient value is reserved, and the non-important coefficients which can meet scarcity can be measured and reconstructed by using lower measurement ration. The design framework of the improved algorithm is shown in Fig. (2).

The important coefficients in DCT coefficient reserve most low-frequency energy of the original image block. In order to make DCT transformation efficient and make hardware implementation easy, the paper improves the block size to be $8 \times 8$. For the practicability of the system, there are 8 important coefficients, and the number of non-important coefficients is determined according to measurement ration. If the measurement ratio is 0.2 , the line number $M$ of random matrix which is selected for the improved method can be computed as follows.

$M=\lfloor 8 \times 8 \times 0.2-8\rfloor=4$

After random matrix measurement, for 56 non-important coefficients, only 4 measurement values and 8 important coefficients are reserved. The real measurement proportion is only $12 / 64=0.1875$. In order to verify the validity of the algorithm and determine the algorithm to be implemented on hardware platform, the paper tests the relationship between reconstruction performance and time consumption of Lena grey image with the resolution ration of $512 \times 512$ under different measurement rations. The initial experimental results are shown in Table 2 . We can see from the table that compared with the basic BCS method, the improved BCS method in the paper has evident advantage for reconstruction image quality and system consumption under low measurement ratio. When the measurement ratio is only 0.2 , the method of improved method for $16 \times 16$ block reduces $84.64 \%$ of system consumption, and the performance benefit is from inhomogeneous measurement and reconstruction scheme. The initial experimental results indicate that the improved method is suitable for real-time hardware image acquisition and compressed system, and the performance is better than the original method.

\section{OPTIMZATION AND IMPLEMENTATION OF ALGORITHM ON DSP PLATFORM}

\subsection{Selection of Core Chips}

TMS320C6455 is a popular high-speed common DSP chip in recent years. The signal processing ability is powerful, and the power consumption and cost is low, so it is 
Table 2. Experimental results of Lena image under improved BCS method.

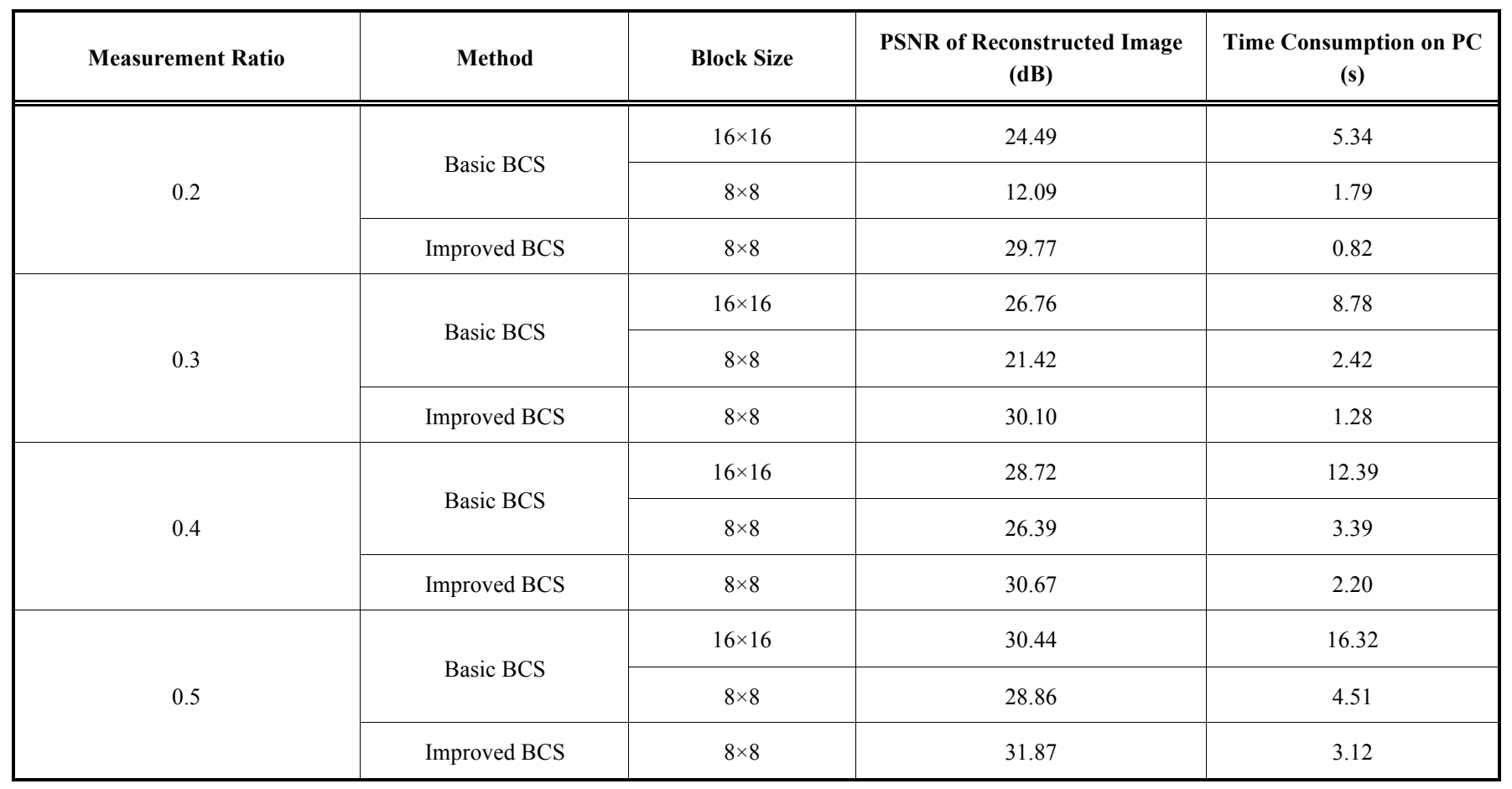

applied by the system needing lots of operation resources $[16,17]$. The characteristics are that the structure is Very Long Instruction Word, it can execute 832 bit instructions for every clock period, and the highest time frequency can reach $1.2 \mathrm{GHz}$, which makes the processing ability of 16 bit reaches 9600MIPS (Million Instructions Per Second) [18]. Compared with the early fixed-point DSP, the on-chip and high-speed capacity of the chip reaches $2096 \mathrm{~K}$ bytes. In addition, C6455 supports external storage interface EMIF and high-speed serial interface SRIO, which not only makes it easy to integrate with other DSP or FPGA, but also can extend DDR2 external memory [19]. The characteristics make the DSP suitable to process image/video signals with large data volume.

\subsection{Implementation and Optimization Method of Algo- rithm}

Image compressed sensing algorithm not only needs large computation resources, but also has high requirement on platform for complexity and storage, and the structure can't be directly implemented on DSP. After determining the framework of the algorithm to be transplanted, algorithm implementation and optimization includes algorithm implementation of DSP and optimization of core code, as follows.

\subsubsection{Floating-point Algorithm fixed-point}

The original algorithm of image compressed sensing is the floating-point algorithm which is implemented on Matlab platform, and the objective platform TMS320C6455 is the fixed-point DSP, which needs to rewrite the original algorithm to be $\mathrm{C}$ language fixed-point source code which can be executed on DSP platform. In fixed-point DSP, the operand generally uses an integer such as C6455 supports the integral data of 8/16/32/40bit. Fixed-point process on core cycling part of the original algorithm can improve the operation efficiency of the algorithm. We can see from Fig. (2) that the original algorithm can be divided into four stages, image transformation, inhomogeneous measurement, sparse-domain reconstruction and image inverse transformation. Transformation and inverse transformation needs to be implemented by integral DCT and inhomogeneous measurement and sparse-domain reconstruction needs to receive fixed point.

Calibration of the number of floating points is the necessary step of floating point and fixed point data transformation [20]. The transformation of floating point number $x$ and fixed-point number y is shown in Formula (7).

$y=$ (int) $x \times 2^{\mathrm{Q}}, x=$ (float) $y \times 2^{-Q}$

The range of $Q$ value should be in the dynamic range of data in operation. Under the premise of avoiding data overflow, Q value with accurate operation should be ensured. After examination, the error of fixed-point algorithm and the original algorithm is less than 10-4. And the random measurement strategy has error, so the accuracy completely meets the requirement of procedure transplant.

\subsubsection{DSP Platform Algorithm Structure Design}

After fix-point algorithm, the processing flow needs to be adjusted according to hardware structure of DSP system. The 


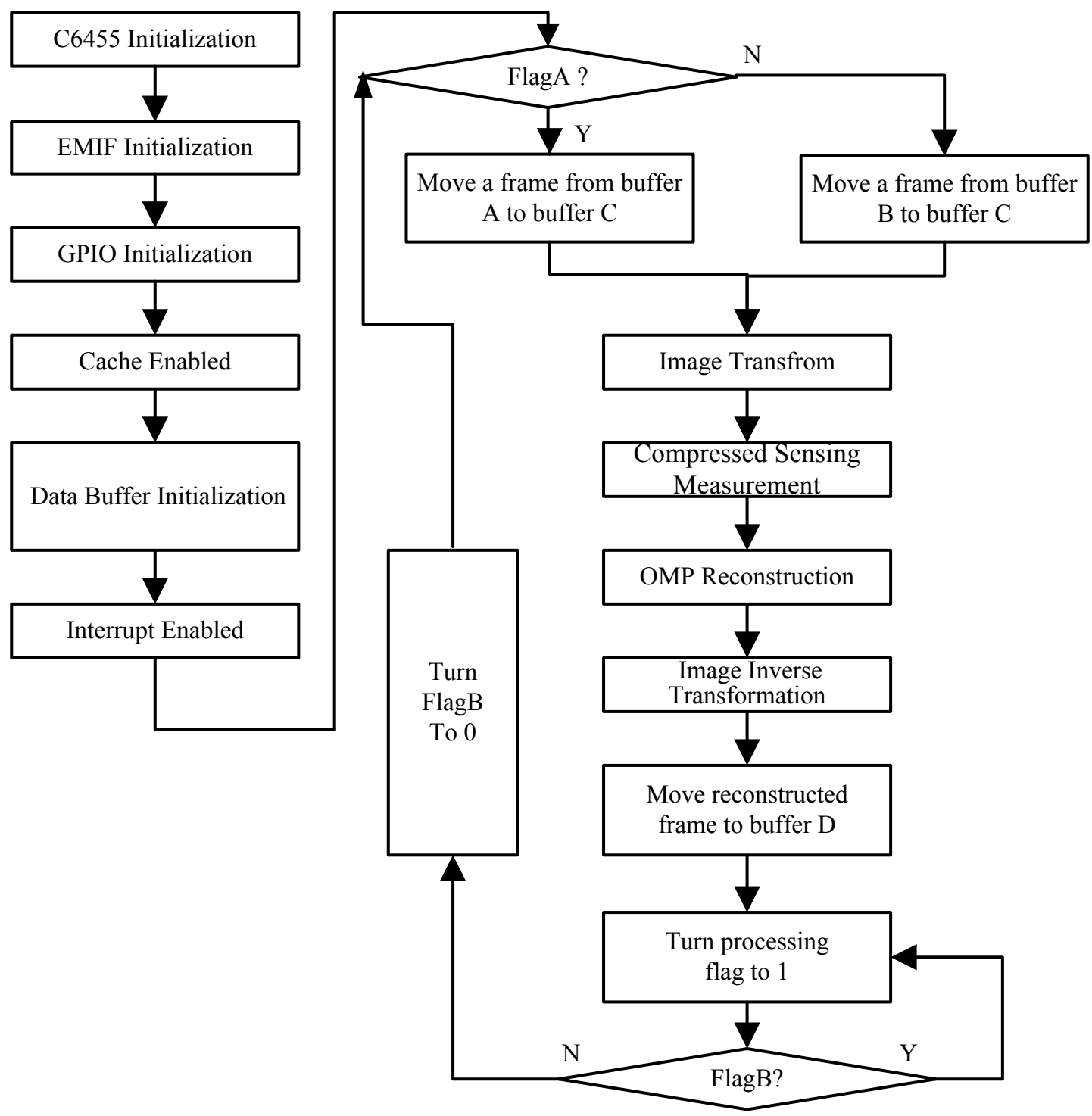

Fig. (3). DSP platform algorithm structure.

structure of the platform algorithm in the paper is shown in Fig. (3). The onboard hardware resource is initially configured including C6455 kernel, EMIF interface, GPIO, initialization of buffering area and Cache. In order to realize rapid exchange and data transmission between algorithm and external equipment, the system uses double buffering mechanism for ping-pong operation. The algorithm structure defines four fixed buffering areas. Buffering area A and buffering area $\mathrm{B}$ is used for ping-pong operation, buffering area $\mathrm{C}$ is used to store an input image, and buffering area D is used to store reconstructed image. And three flag bits are defined including FlagA, FlagB and Status. Flag A is used to describe if data is cached in buffering area $A$ or buffering area B. Flag B is used to describe if the transmission of reconstructed images ends. Status is used to process if the algorithm of an image ends. Two image data frame counters are defined. As the size of input image and reconstructed image may be greater than the data volume of DSP and external equipment, each completed transmission can be realized by the movement between them. Input and output image frame counter is used to compute the movement times of input and output. The structure design of DSP platform algorithm in the paper not only completely meets the time consumption of core processing modules, but also can make it easy to realize high-speed transmission Combined with image frame counter, the input, algorithm process and output of high-speed image data is realized by the way of EDMA transmission.

\subsubsection{DSP Platform Code Optimization}

Improving the operation efficiency of transportation program needs to consider the buffering structure and parallelism of DSP, and demands to fully use flowing function of software, which makes DSP, complete the algorithm with the 
Table 3. Image reconstruction time comparison.

\begin{tabular}{|c|c|c|c|}
\hline & $\begin{array}{c}\text { Basic BCS (16x16)PC Platform Time } \\
\text { Consumption (Second) }\end{array}$ & $\begin{array}{c}\text { Improved BCS } \\
\text { PC Platform Time Consumption } \\
\text { (Second) }\end{array}$ & $\begin{array}{c}\text { Improved BCS } \\
\text { DSPstem Time Consumption } \\
\text { (Second) }\end{array}$ \\
\hline \hline Image & 5.0548 & 0.7867 & 0.1416 \\
\hline Barbara & 5.3642 & 0.8019 & 0.1523 \\
\hline Goldhill & 5.9740 & 0.7981 & 0.1676 \\
\hline
\end{tabular}

shortest time. And it needs to optimize the core cyclic part of the algorithm. The major optimization means include special function optimization, software flow, Cache optimization and compiler configuration.

TI Company provides professional function library and IMG_fdct_8x8 to realize DCT transformation and inverse transformation. The inline functions provided by DSP of C64 series can be used for data combination. 8bit and 16bit data with short word length can be combined to be 32 bit data for process. The software flow needs to be developed to achieve the best DSP software flow performance. After C codes receive the optimization of compiler, the execution efficiency can be achieved, which improves the development speed of the system.

The access speed of DSP to external memory and internal memory has great difference, and the core algorithm should be cached. And the code field and data filed with frequent access should be put into high-speed change to achieve the highest execution and access efficiency. Compared with the original algorithm and the adaptive algorithms in the literatures, the improved algorithm in the paper is suitable for implementing algorithms of DSP platform, the reason for which is that each image block uses unified measurement strategy, and there is no need to change operation parameters in core cycle, which improves the hit rate of high-speed cache. The image block used for the improved algorithm is small, and the required memory space is low, so high-speed cache with $2096 \mathrm{~K}$ byte provided by C6455 platform can meet the requirements of the program.

\section{EXPERIMENT RESULTS AND ANALYSIS}

\subsection{Performance Comparison of Reconstructed Images}

In order to verify the validity of the image compressed sensing method based on C6455 platform, the paper makes algorithm simulation test on four standard static images with the resolution ratio of $512 \times 512$. The objective performance comparison is shown in Fig. (4), which reflects the objective performance of reconstructed images under different measurement scales. From Fig. (4), we can see that the improved BCS algorithm in the paper is better than other algorithms under measurement scales.DSP platform algorithm loses some performance because of fixed point, it can be ignored. The performance under low measurement scale has more important significance compared with compressed sensing algorithm. For example, when the measurement scale is 0.2 , it means that only $1 / 5$ measurement value of the original image sparse domain is used for image reconstruction. We can see from figure 4 that the framework in the paper has evident performance advantage under low measurement scale.

Fig. (5) shows the comparison on subjective performance of Barbara and reconstructed image under the condition that measurement scale is 0.2 . It is evident that difference for the objective performance is only $2 \mathrm{~dB}$, but the subjective quality of the scheme in the paper is evidently higher than that of the original measurement scheme under the condition of low measurement scale.

\subsection{Comparison on Speed of Reconstructing Images}

In order to evaluate the application value of the algorithm on DSP platform, the speed examination is made on PC platform and TMDSDSK6455 platform. The compiling environment of PC platform is Matlab 2013b, and that of DSP platform is CCS v4.0.1. And the measurement scale is 0.2. The comparison for image reconstruction time is shown in Table 3. Compared with the original BCS method, the algorihtm which is implemented on improved DSP platform is more suitable for real-time image process.

\section{CONCLUSION}

This paper firstly analyzes the typical BCS method of image compressed sensing algorithm. For the effect that the algorithm is difficult to be implemented on hardware platform, the paper proposes an inhomogeneous compressed sensing measurement method with low complexity. The improved BCS method uses the same measurement strategy for each image block, which overcomes the disadvantages of the measurement and reconstruction schemes in the literatures, so it is suitable to be implemented on DSP platform with high performance and low cost. The experiment results indicate that the performance and speed of reconstruction images of the improved method is better than that of the basic BCS method. The improved BCS method uses the wide DCT transformation size. And it can easily combine the measurement strategy in the paper and the existing image and video compression scheme, which not only can improve compression efficiency and realize real-time process of hardware, but also can achieve the solution of low-cost DSP platform. 


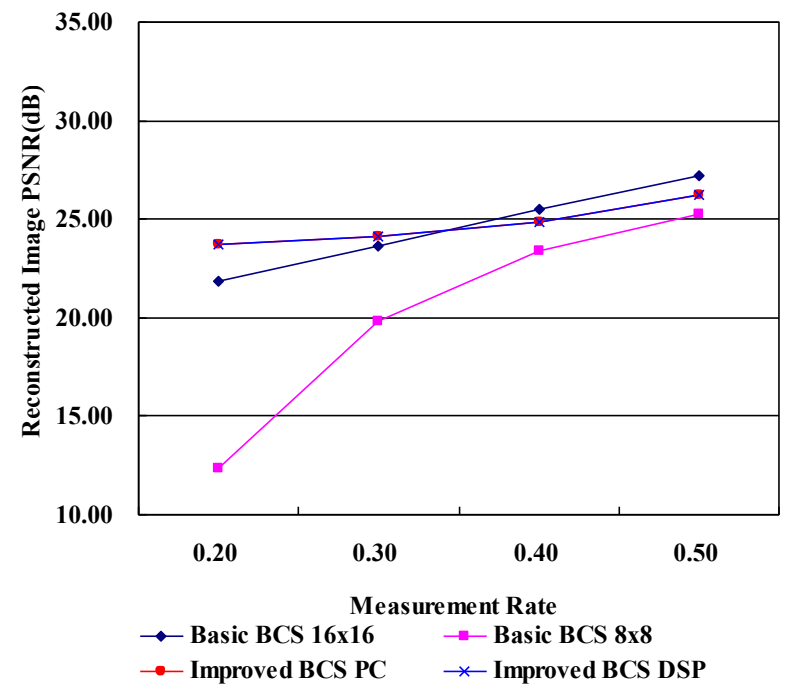

(a) Barbara image reconstruction performance

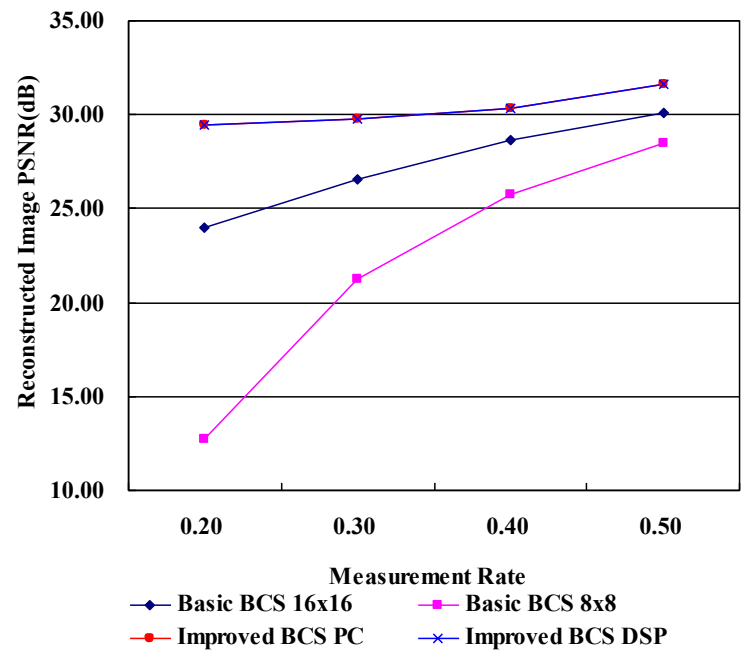

(b) Peppers image reconstruction performance

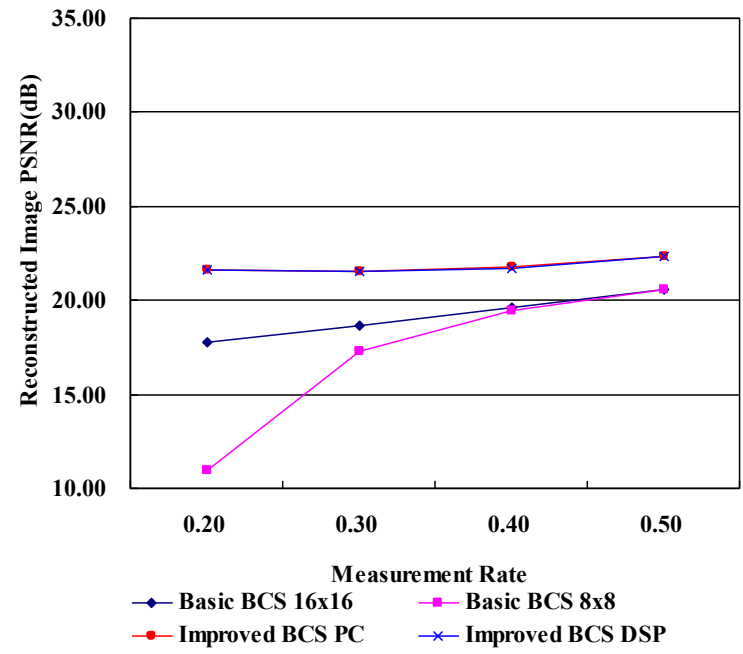

(c) Mandrill image reconstruction performance

Fig. (4). Contd... 


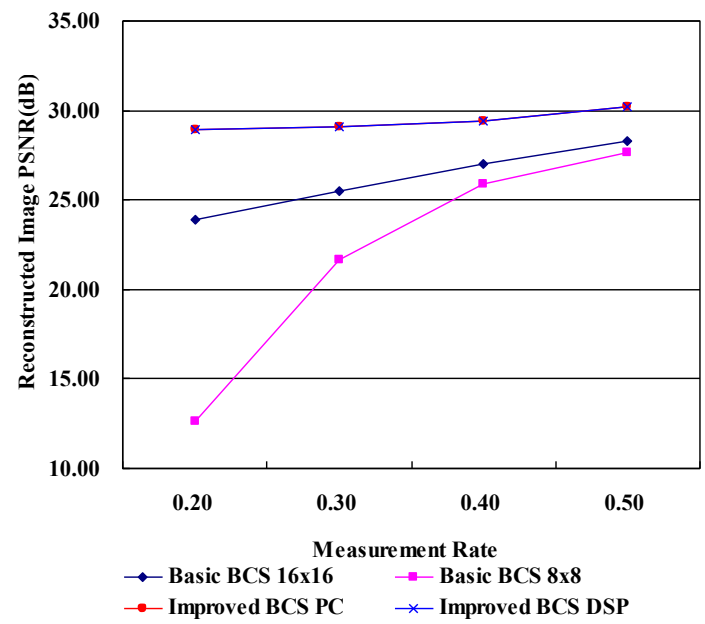

(d) Goldhill image reconstruction performance

Fig. (4). Comparison on objective performance of image reconstruction results.

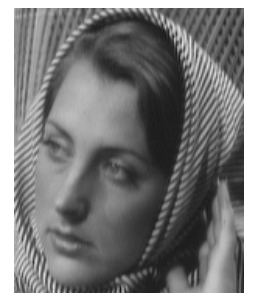

(a) Original image

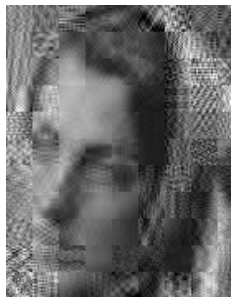

(b) Basic BCS(16x16)

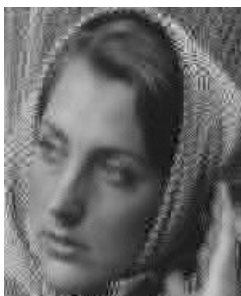

(c) Improved BCS

Fig. (5). Comparison on subjective performance of image reconstruction results.

\section{AUTHOURS PROFILE}

Cheng Zhou was born in Wuhan, April 2nd, 1979. He received the B.S. degree in electronics and information engineering from Hubei University, Wuhan, China, in 2001, and the M.S. and the Ph.D. degrees in pattern recognition and intelligent systems and in control science and control engineering from Huazhong University of Science and Technology, Wuhan, China, in 2007 and 2010, respectively. He is currently an Instructor with the department of electronics and information engineering, South-Central University for Nationalities (SCUEC), Wuhan, China, and he has been engaged in the research of multimedia signal processing, particularly, video coding for MPEG-4, H.264, AVS(China) and HEVC standards since 2004.

Yanhong Lu was born in Hubei, April 30th, 1977. She received the B.S. degree in electronics and information engineering from South-Central University for Nationalities, Wuhan, China, in 1998, and the M.S. degree in communication and information systems from Huazhong University of Science and Technology, Wuhan, China, in 2003. She is currently an Associate Professor with the department of electronics and information Engineering, Wuhan polytechnic, Wuhan, China. Her current research interests include image and video compression.

\section{CONFLICT OF INTEREST}

The authors confirm that this article content has no conflict of interest.

\section{ACKNOWLEDGEMENTS}

Declared none.

\section{REFERENCES}

[1] China News Website, Sike: Global internet flow will reach 56 billion GB in 2013 [EB/OL]. URL at http://news.163.com/10/0326/09/62MLEPHR000146BD.html, vol.3, no.26, 2010

[2] T. Wiegand, G.J. Sullivan, G. Bjøntegaard, and A. Luthra, "Overview of the H.264/AVC video coding standard," IEEE Transactions on Circuits and System for Video Technology, vol. 13, no. 7, pp. 560-576, Jul. 2003.

[3] B. Bross, W. J. Han, G.J. Sullivan, J.R. Ohm, and T. Wiegand, "High Efficiency Video Coding (HEVC) Text Specification Draft 8," Document JCTVC-K1003, ITU-T /ISO/IEC Joint Collaborative Team on Video Coding (JCTVC), 2012.

[4] G.J. Sullivan, J.R. Ohm, W. Han, and T. Wiegand, "Overview of the high efficiency video coding (HEVC) standard," IEEE Transaction on Circuits and Systems for Video Technology, vol. 22, no. 12, pp. 1649-1668, 2012. 
[5] E.J. Candès, J. Romberg, and T. Tao, "Robust uncertainty principles: exact signal reconstruction from highly incomplete frequency information," IEEE Transactions on Information Theory, vol. 52, no. 2, pp. 489-509, 2006.

[6] D. Donoho, "Compressed sensing," IEEE Transactions on Information Theory, vol. 52, no. 4, pp. 1289-1306, 2006.

[7] R. Pan, J. Duan, L. Yu, "Application of compressed sensing to image and video coding," TV Technique, vol. 7, pp. 10-14, 2010.

[8] TMS320C6455 Data book. Texas Instrument Corporation, SPRS276M, pp. 1-10, 2005

[9] L. Gan, "Block compressed sensing of natural images," DSP 2007 15th International Conference on Digital Signal Processing, Cardiff: DSP, pp. 403-406, 2007.

[10] H. Rauhut, "Circulant and Toeplitz matrices in compressed sensing," In: Processing SPAR'09(Saint Malo), 2009.

[11] J. Haupt, and R. Nowak, "Compressive sampling for signal detection," In: Proceedings of the IEEE International Conference on Acoustics, Speech and Signal Processing, pp.1509-1512, 2007.

[12] J. Tropp, and A. Gilbert, "Signal recovery from random measurements via orthogonal matching pursuit," IEEE Transactions on Information Theory, vol. 53, no. 12, pp. 4655-4658, 2007.

[13] C. Zhou, C. Xiong, R. Mao, and J. Gong, "Compressed sensing of images using nonuniform sampling," In: Proceedings of the $4^{\text {th }}$ In- ternational Conference on Intelligent Computation Technology and Automation (ICICTA'11), vol. 2, pp. 483-486, March 2011.

[14] Yi Yang, O.C. Au, F. Lu, W. Xing, and W. Tang "Reweighted compressive sampling for image Compression," PCS Picture Coding Symposium, Chicago, pp. 1-4, 2009.

[15] Y. Yu, B. Wang, and L. Zhang "Saliency-based compressive sampling for image signals," IEEE Signal Processing Letters, vol. 17, no. 11, pp. 973-976, 2010.

[16] Q. Wang, T. Zhang, and R. Zhang, "Implementation of AVS video coding algorithm on TMS320C6455," Data Acquisition and Process, vol. 27, no. 6, pp. 724-729, 2012

[17] J. Song, S. Zhan, L. Ma and F. Yang, "The optimizing implementation of fast loop filter based on C6455 DSP," In: International Conference on E-Business and E-Government(ICEE), pp.4894-4897, 2010.

[18] Q. Zeng, Y. Li, and Z. Ji, "DSP reconstruction method of PET image," Data Acquisition and Process, vol. 28, no. 5, pp. 633-637, 2013.

[19] Texas Instruments.TMS320C6455 fixed-point digital signal processor [EB/OL]. 2009. http://www.ti.com.

[20] L. Chen, J. Yang, and X. Zhang, "Real-time DSP Implementation of Signal Processing Algorithm," Electronic Industry Press: Beijing, pp. 62-64, 2008.

(C) Zhou and Lu; Licensee Bentham Open.

This is an open access article licensed under the terms of the Creative Commons Attribution Non-Commercial License (http://creativecommons.org/licenses/by-nc/3.0/) which permits unrestricted, non-commercial use, distribution and reproduction in any medium, provided the work is properly cited. 